J. Appl. Numer. Optim. 3 (2021), No. 3, pp. 489-499

Available online at http://jano.biemdas.com

https://doi.org/10.23952/jano.3.2021.3.04

\title{
NEW LINEAR CONVERGENCE RESULTS ON QUASI-VARIATIONAL INEQUALITIES
}

\author{
YEKINI SHEHU \\ Department of Mathematics, Zhejiang Normal University, Jinhua 321004, China
}

\begin{abstract}
We propose a new projection algorithm with alternated inertial extrapolation steps, and obtain the linear convergence of the algorithm for a quasi-variational inequality problem in Hilbert spaces. We investigate the cases of under-relaxation and over-relaxation of the proposed algorithm, and give linear convergence results in each case. We also obtain some priori and apriori error bounds of our proposed algorithm.
\end{abstract}

Keywords. Alternated inertial; Error bounds; Linear convergence; Quasi-variational inequalities.

\section{INTRODUCTION}

In this paper, we study the Quasi-Variational Inequality (QVI) problem, which is an extension of the classical Variational Inequality (VI) problem of Fichera [1, 2] and Stampacchia [3] (see also Kinderlehrer and Stampacchia [4]). In a QVI, the associated feasible set of the problem is not fixed, but varies according to some explicit or implicit rule. For example, in many applications, the feasible set is defined as a "moving set", known as a core set, that is shifted by another single-valued mapping; see, e.g., $[5,6,7]$ and the references therein. In such setting, the problem is often called "moving set" QVI.

There exist many techniques for solving QVIs; see, e.g., [8, 9, 10, 11, 12, 13] and the references therein. In 2018, Antipin et al. [5] presented gradient projection and extragradient methods for solving QVIs under the assumptions of strong monotonicity and Lipschitz continuity of the associated mappings. The main disadvantage of the extragradient method, with respect to the classical gradient projection algorithm, is that the number of orthogonal projections and mapping evaluations is doubled per each iteration. For VIs, the extra projection and evaluation in each iteration of the extragradient method guarantee the convergence under weaker assumptions than the strong monotonicity of the associated mapping, however, this is not for QVIs. Thus the extragradient method does not have any advantage over the gradient projection method. A more general gradient projection method with the strong convergence for solving QVIs in real Hilbert spaces was introduced by Mijajlović et al. [14]. This method is efficient in various real applications.

In the field of continuous optimization, inertial-type algorithms attracted much interest in recent years, which is mainly due to their convergence properties. The inertial idea is derived

E-mail address: yekini.shehu @ zjnu.edu.cn.

Received August 9, 2021; Accepted November 12, 2021.

(C)2021 Journal of Applied and Numerical Optimization 
from the field of second order dissipative dynamical systems [15, 16]. It was shown that the algorithms with inertial terms speed up the convergence rate of the original algorithms; see, e.g., the inertial proximal point algorithm [17, 18, 19], the inertial forward-backward splitting method [20, 21, 22], the inertial Douglas-Rachford splitting method [23, 24], the inertial ADMM [25, 26], and the inertial forward-backward-forward method [27].

Motivated by the above results, we aim to present a new inertial type algorithm for solving QVIs. Our highlights are as follows.

(1) We study under-relaxation and over-relaxation approaches of a projection algorithm with alternated inertial steps for quasi-variational inequalities in real Hilbert spaces and obtain linear convergence results.

(2) We present error bounds in terms of priori and apriori error estimates for our algorithm.

The outline of the paper is as follows. In Section 2, we recall some basic facts, definitions, and lemmas, which are needed in the sequel. We present our algorithm and show its convergence analysis in Section 3. The concluding remarks are given in in Section 4, the last section.

\section{PRELIMINARIES}

Let $\mathscr{H}$ be a real Hilbert space with inner product $\langle\cdot, \cdot\rangle$ and norm $\|\cdot\|$. Let $K$ be a nonempty, closed, and convex subset of $\mathscr{H}$. Let $\mathscr{F}: \mathscr{H} \rightarrow \mathscr{H}$ be a nonlinear operator and let $\mathscr{K}: \mathscr{H} \rightrightarrows$ $\mathscr{H}$ be a set-valued mapping, which associates, for any element $u \in \mathscr{H}$, a closed and convex set $\mathscr{K}(u) \subset \mathscr{H}$. With the above data, we are concerned with the following Quasi-Variational Inequality (QVI), which consists of finding a point $u^{*} \in \mathscr{H}$ such that $u^{*} \in \mathscr{K}\left(u^{*}\right)$ and

$$
\left\langle\mathscr{F}\left(u^{*}\right), v-u^{*}\right\rangle \geq 0, \quad \forall v \in \mathscr{K}\left(u^{*}\right) .
$$

Clearly, if $\mathscr{K}(u) \equiv K$ for all $u \in \mathscr{H}$, then the problem is reduced to the classical variational inequality, that is, find a point $u^{*} \in K$ such that

$$
\left\langle\mathscr{F}\left(u^{*}\right), v-u^{*}\right\rangle \geq 0, \quad \forall v \in K .
$$

Definition 2.1. Let $T: \mathscr{H} \rightarrow \mathscr{H}$ be a given mapping.

- $T$ is said to be L-Lipschitz continuous $(L>0)$ if

$$
\|F(x)-F(y)\| \leq L\|x-y\|, \quad \forall x, y \in \mathscr{H} .
$$

- $T$ is said to be $\mu$-strongly monotone $(\mu>0)$ if

$$
\langle T(x)-T(y), x-y\rangle \geq \mu\|x-y\|^{2}, \quad \forall x, y \in \mathscr{H} .
$$

For each point $x \in \mathscr{H}$, there exists a unique nearest point in $K$, denoted by $P_{K}(x)$, such that

$$
\left\|x-P_{K}(x)\right\| \leq\|x-y\|, \quad \forall y \in K .
$$

The mapping $P_{K}: \mathscr{H} \rightarrow K$ is called the metric projection of $\mathscr{H}$ onto $K$, and is characterized [28] by the following two properties:

$$
P_{K}(x) \in K,
$$

and

$$
\left\langle x-P_{K}(x), P_{K}(x)-y\right\rangle \geq 0 \text { for all } x \in \mathscr{H}, y \in K .
$$

If $K$ is a hyper-plane, then (2.2) becomes an equality. 
The following result, which was proved in [29], gives sufficient conditions for the existence of solutions of (2.1).

Lemma 2.1. Let $\mathscr{F}: \mathscr{H} \rightarrow \mathscr{H}$ be L-Lipschitz continuous and $\mu$-strongly monotone on $\mathscr{H}$, and let $\mathscr{K}(\cdot)$ be a set-valued mapping with nonempty, closed, and convex values such that there exists $\lambda \geq 0$ such that

$$
\left\|P_{\mathscr{K}(x)}(z)-P_{\mathscr{K}(y)}(z)\right\| \leq \lambda\|x-y\|, x, y, z \in \mathscr{H}, \quad \lambda+\sqrt{1-\frac{\mu^{2}}{L^{2}}}<1 .
$$

Then QVI (2.1) has a unique solution.

The following result is a known fixed point formulation characterizing the solutions of QVI (2.1).

Lemma 2.2. Let $K(\cdot)$ be a set-valued mapping with nonempty, closed, and convex values in $\mathscr{H}$. Then $x^{*} \in \mathscr{K}\left(x^{*}\right)$ is a solution of QVI (2.1) if and only if, for any $\gamma>0$, it holds that

$$
x^{*}=P_{\mathscr{K}\left(x^{*}\right)}\left(x^{*}-\gamma \mathscr{F}\left(x^{*}\right)\right) .
$$

\section{MAin Results}

We present our main contributions of this paper in this section. First, we introduce our algorithm below.

Algorithm $3.1(\mathrm{H})$. Step 0. Let $x_{0}, x_{1} \in \mathscr{H}$.

Step n. Given the current iterates $x_{n-1}$ and $x_{n}(n \geq 1), \theta \in[0,1)$, and $\alpha_{n} \in(0,2)$, compute

$$
y_{n}= \begin{cases}x_{n}, & n=\text { even } \\ x_{n}+\theta\left(x_{n}-x_{n-1}\right), & n=\text { odd }\end{cases}
$$

and

$$
x_{n+1}=\left(1-\alpha_{n}\right) y_{n}+\alpha_{n} P_{\mathscr{K}\left(y_{n}\right)}\left(y_{n}-\gamma \mathscr{F}\left(y_{n}\right)\right) .
$$

If $y_{n}=P_{\mathscr{K}\left(y_{n}\right)}\left(y_{n}-\gamma \mathscr{F}\left(y_{n}\right)\right)$, then stop and $y_{n}$ is a solution. Otherwise, set $n \leftarrow n+1$ and go to 2.

Algorithm 3.1 is much general for solving quasi-variational inequalities. Indeed, one has the following observations.

Remark 3.1. (a) If $\alpha_{n}=1$ and $\theta=0$ in Algorithm 3.1, then we obtain the standard gradienttype method in [30].

(b) I $\theta=0$ and $0<\alpha_{n} \leq 1$ in Algorithm 3.1, then we obtain [14, Algorithm 1].

(c) If $\theta=0$ and $\alpha_{n}=1$ in Algorithm 3.1, we obtain the proposed method in [5, Eq. (5)] (which was also studied in $[6,30,31,32])$.

Specially, we present the linear convergence analysis of Algorithm 3.1 alongside the associated error bounds. 
3.1. The under-relaxation approach. We first give the convergence analysis of the underrelaxation type of our alternated inertial projection Algorithm 3.1.

Theorem 3.1. Consider the QVI (2.1) with $\mathscr{F}$ being $\mu$-strongly monotone and L-Lipschitz continuous, and assume there exists $\lambda \geq 0$ such that (2.3) holds. Define $\beta:=\sqrt{1-2 \mu \gamma+\gamma^{2} L^{2}}+$ $\lambda \in(0,1)$. Let $\left\{x_{n}\right\}$ be a sequence generated by Algorithm 3.1 with $\theta \in\left[0, \frac{1-\beta}{1+\beta}\right)$ and $0 \leq$ $\frac{2 \theta}{(1+\theta)(1-\beta)}<\alpha \leq \alpha_{n} \leq 1$. In addition, assume that $\gamma \geq 0$ satisfies

$$
\left|\gamma-\frac{\mu}{L^{2}}\right|<\frac{\sqrt{\mu^{2}-L^{2} \lambda(2-\lambda)}}{L^{2}} .
$$

Then $\left\{x_{n}\right\}$ converges linearly to the unique solution $x^{*} \in \mathscr{K}\left(x^{*}\right)$ of $Q V I$ (2.1).

Proof. We divide the proof into two cases.

Case I. Consider $\theta=0$.

In this case, Algorithm 3.1 reduces to [14, Algorithm 1]. Following the same lines of arguments in [14, Theorem 3], we have

$$
\left\|x_{n+1}-x^{*}\right\| \leq\left(1-\alpha_{n}(1-\beta)\right)\left\|x_{n}-x^{*}\right\| \leq(1-\alpha(1-\beta))\left\|x_{n}-x^{*}\right\| .
$$

Note that $1-\alpha(1-\beta) \in(0,1)$ due to $\alpha \in(0,1]$ and $1-\beta \in(0,1)$. Consequently, we obtain by induction that

$$
\left\|x_{n+1}-x^{*}\right\| \leq(1-\alpha(1-\beta))^{n}\left\|x_{1}-x^{*}\right\| .
$$

Case II. Consider $\theta \in\left(0, \frac{1-\beta}{1+\beta}\right)$.

Note that

$$
x^{*}=\left(1-\alpha_{n}\right) x^{*}+\alpha_{n} P_{\mathscr{K}\left(x^{*}\right)}\left(x^{*}-\gamma \mathscr{F}\left(x^{*}\right)\right) .
$$

From Algorithm 3.1, one has

$$
\begin{aligned}
&\left\|x_{n+1}-x^{*}\right\| \\
& \leq\left\|\left(1-\alpha_{n}\right)\left(y_{n}-x^{*}\right)\right\|+\alpha_{n}\left\|P_{\mathscr{K}\left(y_{n}\right)}\left(y_{n}-\gamma \mathscr{F}\left(y_{n}\right)\right)-P_{\mathscr{K}\left(x^{*}\right)}\left(x^{*}-\gamma \mathscr{F}\left(x^{*}\right)\right)\right\| \\
& \leq\left(1-\alpha_{n}\right)\left\|y_{n}-x^{*}\right\|+\alpha_{n}\left\|P_{\mathscr{K}\left(y_{n}\right)}\left(y_{n}-\gamma \mathscr{F}\left(y_{n}\right)\right)-P_{\mathscr{K}\left(x^{*}\right)}\left(y_{n}-\gamma \mathscr{F}\left(y_{n}\right)\right)\right\| \\
&+\alpha_{n}\left\|P_{\mathscr{K}\left(x^{*}\right)}\left(y_{n}-\gamma \mathscr{F}\left(y_{n}\right)\right)-P_{\mathscr{K}\left(x^{*}\right)}\left(x^{*}-\gamma \mathscr{F}\left(x^{*}\right)\right)\right\| \\
& \leq\left(1-\alpha_{n}\right)\left\|y_{n}-x^{*}\right\|+\alpha_{n} \lambda\left\|y_{n}-x^{*}\right\| \\
&+\alpha_{n}\left\|\left(y_{n}-\gamma \mathscr{F}\left(y_{n}\right)\right)-\left(x^{*}-\gamma \mathscr{F}\left(x^{*}\right)\right)\right\| .
\end{aligned}
$$

Since $\mathscr{F}$ is $\mu$-strongly monotone and $L$-Lipschitz continuous, one has

$$
\begin{aligned}
\left\|\left(y_{n}-\gamma \mathscr{F}\left(y_{n}\right)\right)-\left(x^{*}-\gamma \mathscr{F}\left(x^{*}\right)\right)\right\|^{2}= & \left\|y_{n}-x^{*}\right\|^{2}-2 \gamma\left\langle\mathscr{F}\left(y_{n}\right)-\mathscr{F}\left(x^{*}\right), y_{n}-x^{*}\right\rangle \\
& +\gamma^{2}\left\|\mathscr{F}\left(y_{n}\right)-\mathscr{F}\left(x^{*}\right)\right\|^{2} \\
\leq & \left(1-2 \mu \gamma+\gamma^{2} L^{2}\right)\left\|y_{n}-x^{*}\right\|^{2} .
\end{aligned}
$$

Substituting (3.4) into (3.3), we conclude that

$$
\begin{aligned}
& \left\|x_{n+1}-x^{*}\right\| \\
& \leq\left(1-\alpha_{n}\right)\left\|y_{n}-x^{*}\right\|+\alpha_{n} \lambda\left\|y_{n}-x^{*}\right\|+\alpha_{n} \sqrt{1-2 \mu \gamma+\gamma^{2} L^{2}}\left\|y_{n}-x^{*}\right\| \\
& =\left(1-\alpha_{n}(1-\beta)\right)\left\|y_{n}-x^{*}\right\| .
\end{aligned}
$$


As a special case of (3.5), we have

$$
\begin{aligned}
\left\|x_{2 n+2}-x^{*}\right\|^{2} \leq & \left(1-\alpha_{2 n+1}(1-\beta)\right)^{2}\left\|y_{2 n+1}-x^{*}\right\|^{2} \\
= & \left(1-\alpha_{2 n+1}(1-\beta)\right)^{2}\left[(1+\theta)\left\|x_{2 n+1}-x^{*}\right\|^{2}\right. \\
& \left.-\theta\left\|x_{2 n}-x^{*}\right\|^{2}+\theta(1+\theta)\left\|x_{2 n+1}-x_{2 n}\right\|^{2}\right] \\
= & q_{2 n+1}^{2}\left[(1+\theta)\left\|x_{2 n+1}-x^{*}\right\|^{2}-\theta\left\|x_{2 n}-x^{*}\right\|^{2}\right. \\
& \left.+\theta(1+\theta)\left\|x_{2 n+1}-x_{2 n}\right\|^{2}\right],
\end{aligned}
$$

where $q_{n}:=1-\alpha_{n}(1-\beta), \forall n \geq 1$. From (3.5) and (3.6), we have

$$
\begin{aligned}
\left\|x_{2 n+2}-x^{*}\right\|^{2} \leq & q_{2 n+1}^{2}\left[(1+\theta)\left\|x_{2 n+1}-x^{*}\right\|^{2}-\theta\left\|x_{2 n}-x^{*}\right\|^{2}+\theta(1+\theta)\left\|x_{2 n+1}-x_{2 n}\right\|^{2}\right] \\
\leq & q_{2 n+1}^{2}\left[q_{2 n}^{2}(1+\theta)\left\|x_{2 n}-x^{*}\right\|^{2}-\theta\left\|x_{2 n}-x^{*}\right\|^{2}+\theta(1+\theta)\left\|x_{2 n+1}-x_{2 n}\right\|^{2}\right] \\
\leq & q_{2 n+1}^{2}\left[q_{2 n}^{2}(1+\theta)\left\|x_{2 n}-x^{*}\right\|^{2}-\theta\left\|x_{2 n}-x^{*}\right\|^{2}\right. \\
& \left.+\theta(1+\theta)\left(\left\|x_{2 n+1}-x^{*}\right\|+\left\|x_{2 n}-x^{*}\right\|\right)^{2}\right] \\
\leq & q_{2 n+1}^{2}\left[q_{2 n}^{2}(1+\theta)\left\|x_{2 n}-x^{*}\right\|^{2}-\theta\left\|x_{2 n}-x^{*}\right\|^{2}\right. \\
& \left.+\theta(1+\theta)\left(1+q_{2 n}\right)^{2}\left\|x_{2 n}-x^{*}\right\|^{2}\right] \\
\leq & q_{2 n+1}^{2}\left\|x_{2 n}-x^{*}\right\|^{2},
\end{aligned}
$$

where

due to $\frac{2 \theta}{(1+\theta)(1-\beta)}<\alpha_{n}$. By (3.7), we obtain

$$
q_{2 n}^{2}(1+\theta)-\theta+\theta(1+\theta)\left(1+q_{2 n}\right)^{2}<1
$$

$$
\begin{aligned}
\left\|x_{2 n+2}-x^{*}\right\| & \leq q_{2 n+1}\left\|x_{2 n}-x^{*}\right\| \\
& \leq \frac{1-\theta}{1+\theta}\left\|x_{2 n}-x^{*}\right\| \\
& \vdots \\
& \leq\left(\frac{1-\theta}{1+\theta}\right)^{n}\left\|x_{2}-x^{*}\right\| .
\end{aligned}
$$

This implies that

$$
\left\|x_{2 n}-x^{*}\right\| \leq\left(\frac{1-\theta}{1+\theta}\right)^{n-1}\left\|x_{2}-x^{*}\right\|
$$

Substituting (3.8) into (3.5), we conclude

$$
\begin{aligned}
\left\|x_{2 n+1}-x^{*}\right\| & \leq q_{2 n}\left\|x_{2 n}-x^{*}\right\| \\
& \leq \frac{1-\theta}{1+\theta}\left\|x_{2 n}-x^{*}\right\| \\
& \leq\left\|x_{2 n}-x^{*}\right\| \\
& \leq\left(\frac{1-\theta}{1+\theta}\right)^{n}\left\|x_{2}-x^{*}\right\| .
\end{aligned}
$$


In view of (3.8) and (3.9), we obtain that $\left\{x_{n}\right\}$ converges $R$-linearly to $x^{*}$. This concludes the proof.

If $\mathscr{K}(x), x \in \mathscr{H}$ is a "moving set", i.e., the case that $\mathscr{K}(x):=c(x)+K, x \in \mathscr{H}$, where $c: \mathscr{H} \rightarrow \mathscr{H}$ is a $\lambda$-Lipschitz continuous mapping and $K \subset \mathscr{H}$ is a nonempty, closed, and convex subset. Then (2.3) holds with the same value of $\lambda$ (see [30]). From Theorem 3.1, we have the following corollary.

Corollary 3.1. Consider the QVI (2.1) with $\mathscr{F}$ being $\mu$-strongly monotone and L-Lipschitz continuous, and suppose that $\mathscr{K}(x):=c(x)+K, x \in \mathscr{H}$, where $c: \mathscr{H} \rightarrow \mathscr{H}$ is a $\lambda$-Lipschitz continuous mapping and $K$ is a nonempty, closed, and convex subset of $\mathscr{H}$. Let $\left\{x_{n}\right\}$ be a sequence generated by Algorithm 3.1 with $\gamma \geq 0$ satisfying (3.13). Define $\beta:=\sqrt{1-2 \mu \gamma+\gamma^{2} L^{2}}+\lambda \in$ $(0,1)$. Assume that $\theta \in\left[0, \frac{1-\beta}{1+\beta}\right)$ and $0 \leq \frac{2 \theta}{(1+\theta)(1-\beta)}<\alpha \leq \alpha_{n} \leq 1$. Then $\left\{x_{n}\right\}$ converges $R$-linearly to the unique solution $x^{*} \in \mathscr{K}\left(x^{*}\right)$ of $Q V I(2.1)$.

Remark 3.2. (a) Theorem 3.1 reduces to [30, Theorem 3.3] if $\alpha_{n}=1$ and $\theta=0$ in Algorithm 3.1.

(b) Theorem 3.1 reduces to [14, Theorem ] if $\theta=0$ and $0<\alpha_{n} \leq 1$ in Theorem 3.1.

(c) Theorem 3.1 reduces to [5, Theorem 3.1] if $\theta=0$ and $\alpha_{n}=1$ in Algorithm 3.1.

The following results give priori and posteriori error estimates of the sequence generated by Algorithm 3.1.

Theorem 3.2. Let all the assumptions in Theorem 3.1 be satisfied. Let $\theta=0$ in Algorithm 3.1 and $\left\{x_{n}\right\}$ be generated by Algorithm 3.1. Then

$$
\left\|x_{n+1}-x^{*}\right\| \leq(1-\alpha(1-\beta))^{n} \frac{1}{\alpha(1-\beta)}\left\|x_{2}-x_{1}\right\|, \forall n \geq 1
$$

and

$$
\left\|x_{n+1}-x^{*}\right\| \leq \frac{1-\alpha(1-\beta)}{(1-\beta) \alpha}\left\|x_{n}-x_{n+1}\right\|, \forall n \geq 1
$$

Proof. From (3.1), one has

$$
\begin{aligned}
\left\|x_{n}-x^{*}\right\| & \leq\left\|x_{n}-x_{n+1}\right\|+\left\|x_{n+1}-x^{*}\right\| \\
& \leq\left\|x_{n}-x_{n+1}\right\|+\left(1-\alpha_{n}(1-\beta)\right)\left\|x_{n}-x^{*}\right\| .
\end{aligned}
$$

It follows that

$$
\left\|x_{n}-x^{*}\right\| \leq \frac{1}{\alpha_{n}(1-\beta)}\left\|x_{n}-x_{n+1}\right\| .
$$

By (3.2) and (3.10), we obtain

$$
\begin{aligned}
\left\|x_{n+1}-x^{*}\right\| & \leq(1-\alpha(1-\beta))^{n}\left\|x_{1}-x^{*}\right\| \\
& \leq(1-\alpha(1-\beta))^{n} \frac{1}{\alpha_{1}(1-\beta)}\left\|x_{2}-x_{1}\right\| \\
& \leq(1-\alpha(1-\beta))^{n} \frac{1}{\alpha(1-\beta)}\left\|x_{2}-x_{1}\right\| .
\end{aligned}
$$


In view of (3.1) and (3.10), we have

$$
\begin{aligned}
\left\|x_{n+1}-x^{*}\right\| & \leq\left(1-\alpha_{n}(1-\beta)\right)\left\|x_{n}-x^{*}\right\| \\
& \leq \frac{1-\alpha_{n}(1-\beta)}{\alpha_{n}(1-\beta)}\left\|x_{n}-x_{n+1}\right\| .
\end{aligned}
$$

Observe that

$$
\frac{1-\alpha_{n}(1-\beta)}{\alpha_{n}(1-\beta)} \leq \frac{1-\alpha(1-\beta)}{\alpha(1-\beta)}
$$

due to $\alpha<\alpha_{n}$. This implies from (3.11) that

$$
\left\|x_{n+1}-x^{*}\right\| \leq \frac{1-\alpha(1-\beta)}{\alpha(1-\beta)}\left\|x_{n}-x_{n+1}\right\|
$$

Theorem 3.3. Let all assumptions in Theorem 3.1 be satisfied. Let $\left\{x_{n}\right\}$ be generated by Algorithm 3.1. Then, for $\theta \in\left(0, \frac{1-\beta}{1+\beta}\right)$,

(i)

$$
\left\|x_{2 n+2}-x^{*}\right\| \leq\left(\frac{1-\theta}{1+\theta}\right)^{n}\left(\frac{1+\theta}{2 \theta}\right)\left\|x_{2}-x_{4}\right\|, \forall n \geq 1
$$

and

$$
\left\|x_{2 n+2}-x^{*}\right\| \leq\left(\frac{1-\theta}{1+\theta}\right)\left(\frac{1+\theta}{2 \theta}\right)\left\|x_{2 n}-x_{2 n+2}\right\|, \forall n \geq 1
$$

(ii)

and

$$
\left\|x_{2 n+1}-x^{*}\right\| \leq\left(\frac{1-\theta}{1+\theta}\right)^{n-1}\left(\frac{1+\theta}{2 \theta}\right)\left\|x_{2}-x_{4}\right\|, \forall n \geq 1
$$

$$
\left\|x_{2 n+1}-x^{*}\right\| \leq\left(\frac{1-\theta}{1+\theta}\right)\left(\frac{1+\theta}{2 \theta}\right)\left\|x_{2 n}-x_{2 n+2}\right\|, \forall n \geq 1 .
$$

Proof. Observe that

$$
\begin{aligned}
\left\|x_{2 n}-x^{*}\right\| & \leq\left\|x_{2 n}-x_{2 n+2}\right\|+\left\|x_{2 n+2}-x^{*}\right\| \\
& \leq\left\|x_{2 n}-x_{2 n+2}\right\|+\left(\frac{1-\theta}{1+\theta}\right)\left\|x_{2 n}-x^{*}\right\| .
\end{aligned}
$$

Therefore,

$$
\left\|x_{2 n}-x^{*}\right\| \leq\left(\frac{1+\theta}{2 \theta}\right)\left\|x_{2 n}-x_{2 n+2}\right\| .
$$

From (3.8) and (3.12), we conclude

$$
\begin{aligned}
\left\|x_{2 n+2}-x^{*}\right\| & \leq\left(\frac{1-\theta}{1+\theta}\right)^{n}\left\|x_{2}-x^{*}\right\| \\
& \leq\left(\frac{1-\theta}{1+\theta}\right)^{n}\left(\frac{1+\theta}{2 \theta}\right)\left\|x_{2}-x_{4}\right\| .
\end{aligned}
$$

Using (3.8) and (3.12) again, we arrive at

$$
\begin{aligned}
\left\|x_{2 n+2}-x^{*}\right\| & \leq\left(\frac{1-\theta}{1+\theta}\right)\left\|x_{2 n}-x^{*}\right\| \\
& \leq\left(\frac{1-\theta}{1+\theta}\right)\left(\frac{1+\theta}{2 \theta}\right)\left\|x_{2 n}-x_{2 n+2}\right\| .
\end{aligned}
$$


Hence, (i) is established.

In view of (3.8), we have

$$
\left(\frac{1-\theta}{1+\theta}\right)\left\|x_{2 n}-x^{*}\right\| \leq\left(\frac{1-\theta}{1+\theta}\right)^{n}\left\|x_{2}-x^{*}\right\| .
$$

Thus

$$
\left\|x_{2 n}-x^{*}\right\| \leq\left(\frac{1-\theta}{1+\theta}\right)^{n-1}\left\|x_{2}-x^{*}\right\| .
$$

Using (3.9) and (3.12), we assert that

$$
\begin{aligned}
\left\|x_{2 n+1}-x^{*}\right\| & \leq\left(\frac{1-\theta}{1+\theta}\right)^{n-1}\left\|x_{2}-x^{*}\right\| \\
& \leq\left(\frac{1-\theta}{1+\theta}\right)^{n-1}\left(\frac{1+\theta}{2 \theta}\right)\left\|x_{2}-x_{4}\right\| .
\end{aligned}
$$

Furthermore, (3.12) implies that

$$
\begin{aligned}
\left\|x_{2 n+1}-x^{*}\right\| & \leq\left(\frac{1-\theta}{1+\theta}\right)\left\|x_{2 n}-x^{*}\right\| \\
& \leq\left(\frac{1-\theta}{1+\theta}\right)\left(\frac{1+\theta}{2 \theta}\right)\left\|x_{2 n}-x_{2 n+2}\right\| .
\end{aligned}
$$

This establishes (ii).

3.2. The over-relaxation approach. In this subsection, we investigate the convergence analysis of the alternated inertial projection Algorithm 3.1 in the case of over-relaxation.

Theorem 3.4. Assume that $\mathscr{F}$ is $\mu$-strongly monotone and L-Lipschitz continuous in QVI (2.1) and there exists $\lambda \geq 0$ such that (2.3) holds. Define $\beta:=\sqrt{1-2 \mu \gamma+\gamma^{2} L^{2}}+\lambda \in(0,1)$. Let $\left\{x_{n}\right\}$ be a sequence generated by Algorithm 3.1 with $\theta \in\left[0, \frac{1-\beta}{1+\beta}\right)$ and $1<\alpha_{n} \leq \alpha^{*}<$ $\frac{2}{(1+\theta)(1+\beta)}$. In addition, assume that $\gamma \geq 0$ satisfies

$$
\left|\gamma-\frac{\mu}{L^{2}}\right|<\frac{\sqrt{\mu^{2}-L^{2} \lambda(2-\lambda)}}{L^{2}} .
$$

Then $\left\{x_{n}\right\}$ converges $R$-linearly to the unique solution $x^{*} \in \mathscr{K}\left(x^{*}\right)$ of $Q V I$ (2.1).

Proof. Following (3.3)-(3.5) above, we have

$$
\begin{aligned}
\left\|x_{n+1}-x^{*}\right\| \leq & \left(\alpha_{n}-1\right)\left\|y_{n}-x^{*}\right\|+\alpha_{n} \lambda\left\|y_{n}-x^{*}\right\| \\
& +\alpha_{n}\left\|\left(y_{n}-\gamma \mathscr{F}\left(y_{n}\right)\right)-\left(x^{*}-\gamma \mathscr{F}\left(x^{*}\right)\right)\right\| \\
\leq & \left(\alpha_{n}-1\right)\left\|y_{n}-x^{*}\right\|+\alpha_{n} \lambda\left\|y_{n}-x^{*}\right\| \\
& +\alpha_{n} \sqrt{1-2 \mu \gamma+\gamma^{2} L^{2}}\left\|y_{n}-x^{*}\right\| \\
= & \left(\alpha_{n}(1+\beta)-1\right)\left\|y_{n}-x^{*}\right\| .
\end{aligned}
$$

We consider the following two cases.

Case I. Consider $\theta=0$.

In this case, we have from (3.14) that

$$
\left\|x_{n+1}-x^{*}\right\| \leq\left(\alpha_{n}(1+\beta)-1\right)\left\|x_{n}-x^{*}\right\| \leq\left(\alpha^{*}(1+\beta)-1\right)\left\|x_{n}-x^{*}\right\| .
$$


Since $1<\alpha^{*}(1+\beta)<2$, one has $\alpha^{*}(1+\beta)-1 \in(0,1)$. It follows that

$$
\left\|x_{n+1}-x^{*}\right\| \leq\left(\alpha^{*}(1+\beta)-1\right)^{n}\left\|x_{1}-x^{*}\right\| .
$$

Case II. Consider $\theta \in\left(0, \frac{1-\beta}{1+\beta}\right)$.

Define $\bar{q}_{n}:=\alpha_{n}(1+\beta)-1$. One concludes from (3.14) that (observe the arguments of (3.6)$(3.7))$

$$
\begin{aligned}
\left\|x_{2 n+2}-x^{*}\right\|^{2} & \leq \bar{q}_{2 n+1}^{2}\left(\bar{q}_{2 n}^{2}(1+\theta)-\theta+\theta(1+\theta)\left(1+\bar{q}_{2 n}\right)^{2}\right)\left\|x_{2 n}-x^{*}\right\| \\
& \leq \bar{q}_{2 n+1}^{2}\left\|x_{2 n}-x^{*}\right\|^{2},
\end{aligned}
$$

since $\bar{q}_{2 n}^{2}(1+\theta)-\theta+\theta(1+\theta)\left(1+\bar{q}_{2 n}\right)^{2}<1$, which is due to $\alpha_{n}<\frac{2}{(1+\theta)(1+\beta)}$ and $\theta<\frac{1-\beta}{1+\beta}$. Observe that

$$
\bar{q}_{n} \leq \alpha^{*}(1+\beta)-1 \leq(1+\beta) \frac{2}{(1+\theta)(1+\beta)}-1=\frac{1-\theta}{1+\theta} .
$$

So,

$$
\begin{aligned}
\left\|x_{2 n+2}-x^{*}\right\| & \leq \bar{q}_{2 n+1}\left\|x_{2 n}-x^{*}\right\| \\
& <\frac{1-\theta}{1+\theta}\left\|x_{2 n}-x^{*}\right\| \\
& \vdots \\
& \leq\left(\frac{1-\theta}{1+\theta}\right)^{n}\left\|x_{2}-x^{*}\right\| .
\end{aligned}
$$

This implies that

$$
\left\|x_{2 n}-x^{*}\right\| \leq\left(\frac{1-\theta}{1+\theta}\right)^{n-1}\left\|x_{2}-x^{*}\right\|
$$

Similarly, one has

$$
\begin{aligned}
\left\|x_{2 n+1}-x^{*}\right\| & \leq \bar{q}_{2 n}\left\|y_{2 n}-x^{*}\right\| \\
& <\frac{1-\theta}{1+\theta}\left\|x_{2 n}-x^{*}\right\| \\
& \leq\left\|x_{2 n}-x^{*}\right\| \\
& \leq\left(\frac{1-\theta}{1+\theta}\right)^{n-1}\left\|x_{2}-x^{*}\right\| .
\end{aligned}
$$

Therefore, $\left\{x_{n}\right\}$ converges to $x^{*} R$-linearly.

Corollary 3.2. Consider the QVI (2.1) with $\mathscr{F}$ being $\mu$-strongly monotone and L-Lipschitz continuous and suppose that $\mathscr{K}(x):=c(x)+K, x \in \mathscr{H}$, where $c: \mathscr{H} \rightarrow \mathscr{H}$ is a $\lambda$-Lipschitz continuous mapping and $K$ is a nonempty, closed, and convex subset of $\mathscr{H}$. Define $\beta:=$ $\sqrt{1-2 \mu \gamma+\gamma^{2} L^{2}}+\lambda \in(0,1)$. Let $\left\{x_{n}\right\}$ be a sequence generated by Algorithm 3.1 with $\theta \in$ $\left[0, \frac{1-\beta}{1+\beta}\right)$ and $1<\alpha_{n} \leq \alpha^{*}<\frac{2}{(1+\theta)(1+\beta)}$. Let $\left\{x_{n}\right\}$ be a sequence generated by Algorithm 3.1 with $\gamma \geq 0$ satisfying (3.13). Then $\left\{x_{n}\right\}$ converges $R$-linearly to the unique solution $x^{*} \in \mathscr{K}\left(x^{*}\right)$ of $Q V I(2.1)$. 


\section{FINAL REMARKS}

In this paper, we gave the linear rate convergence analysis of the generalized projected gradient method with alternated inertial steps for solving the quasi-variational inequality in real Hilbert spaces. We considered under-relaxation and over-relaxation cases in our analysis. We also obtained some priori and apriori error estimates of the sequence generated by our algorithm. Many gradient-type methods for solving the quasi-variational inequality in the literature are the special cases of our new algorithm.

\section{REFERENCES}

[1] G. Fichera, Sul problema elastostatico di Signorini con ambigue condizioni al contorno (On the Signorini elastostatic problem with ambiguous boundary conditions), Rendiconti Accademia Lincei, serie 8, 34 (1963), $138-142$.

[2] G. Fichera, Problemi elastostatici con vincoli unilaterali: il problema di Signorini con ambigue condizioni al contorno (Elastostatic problems with unilateral constraints: the Signorini problem with ambiguous boundary conditions), Memorie Accademia Lincei, serie 9,7 (1964), 91-140.

[3] G. Stampacchia, Formes bilineaires coercitives sur les ensembles convexes, Académie des Sciences de Paris 258 (1964), 4413-4416.

[4] D. Kinderlehrer, G. Stampacchia, An Introduction to Variational Inequalities and Their Applications, Academic Press, New York-London, 1980.

[5] A.S. Antipin, M. Jaćimović, N. Mijajlovi, Extragradient method for solving quasivariational inequalities, Optimization 67 (2018), 103-112.

[6] M.A. Noor, An iterative scheme for a class of quasi variational inequalities, J. Math. Anal. Appl. 110 (1985), 463-468.

[7] M. A. Noor, Quasi variational inequalities, Appl. Math. Lett. 1 (1988), 367-370.

[8] D. Aussel, S. Sagratella, Sufficient conditions to compute any solution of a quasivariational inequality via a variational inequality, Math. Methods Oper. Res. 85 (2017), 3-18.

[9] F. Facchinei, C. Kanzow, S. Sagratella, Solving quasi-variational inequalities via their KKT conditions, Math. Program. 144 (2014), 369-412.

[10] V. Latorre, S. Sagratella, A canonical duality approach for the solution of affine quasi-variational inequalities, J. Global Optim. 64 (2016), 433-449.

[11] Y. Shehu, Linear convergence for quasi-variational inequalities with inertial projection-type method, Numer. Funct. Anal. Optim. https://doi.org/10.1080/01630563.2021.1950760.

[12] Y. Shehu, A. Gibali, S. Sagratella, Inertial projection-type methods for solving quasi-variational inequalities in real Hilbert spaces, J. Optim. Theory Appl. 184 (2020), 877-894.

[13] L. V. Nguyen, X. Qin, Some results on strongly pseudomonotone quasi-variational inequalities, Set-Valued Var. Anal. 28 (2020), 239-257.

[14] N. Mijajlović, M. Jaćimović, M. A. Noor, Gradient-type projection methods for quasi-variational inequalities, Optim. Lett. 13 (2019), 1885-1896.

[15] H. Attouch, X. Goudon, P. Redont, The heavy ball with friction. I. The continuous dynamical system, Commun. Contemp. Math. 2 (2000), 1-34.

[16] H. Attouch, M.O. Czarnecki, Asymptotic control and stabilization of nonlinear oscillators with non-isolated equilibria, J. Differential Equations 179 (2002), 278-310.

[17] F. Alvarez, Weak convergence of a relaxed and inertial hybrid projection-proximal point algorithm for maximal monotone operators in Hilbert space, SIAM J. Optim. 14 (2003), 773-782.

[18] F. Alvarez, H. Attouch, An inertial proximal method for maximal monotone operators via discretization of a nonlinear oscillator with damping, Set-Valued Anal. 9 (2001), 3-11.

[19] A. Beck, M. Teboulle, A fast iterative shrinkage-thresholding algorithm for linear inverse problems, SIAM J. Imaging Sci. 2 (2009), 183-202.

[20] H. Attouch, J. Peypouquet, P. Redont, A dynamical approach to an inertial forward-backward algorithm for convex minimization, SIAM J. Optim. 24 (2014), 232-256. 
[21] D. A. Lorenz, T. Pock, An inertial forward-backward algorithm for monotone inclusions, J. Math. Imaging Vision 51 (2015), 311-325.

[22] X. Qin, L. Wang, J.C. Yao, Inertial splitting method for maximal monotone mappings, J. Nonlinear Convex Anal. 21 (2020), 2325-2333.

[23] R.I. Boţ, E.R. Csetnek, C. Hendrich, Inertial Douglas-Rachford splitting for monotone inclusion, Appl. Math. Comput. 256 (2015), 472-487.

[24] Y. Shehu, Convergence rate analysis of inertial Krasnoselskii-Mann-type iteration with applications, Numer. Funct. Anal. Optim. 39 (2018), 1077-1091.

[25] R.I. Boţ, E.R. Csetnek, An inertial alternating direction method of multipliers, Minimax Theory Appl. 1 (2016), 29-49.

[26] C. Chen, R.H. Chan, S. Ma, J. Yang, Inertial proximal ADMM for linearly constrained separable convex optimization, SIAM J. Imaging Sci. 8 (2015), 2239-2267.

[27] R.I. Boţ, E.R. Csetnek, An inertial forward-backward-forward primal-dual splitting algorithm for solving monotone inclusion problems, Numer. Algo. 71 (2016), 519-540.

[28] H. Zhou, X. Qin, Fixed Points of Nonlinear Operator, De Gruyter, Berlin, 2020.

[29] M.A. Noor, W. Oettli, On general nonlinear complementarity problems and quasi equilibria, Matematiche (Catania) 49 (1994), 313-331.

[30] Y. Nesterov, L. Scrimali, Solving strongly monotone variational and quasi-variational inequalities, Discrete Contin. Dyn. Syst. 31 (2011), 1383-1396.

[31] A.S. Antipin, M. Jaćimović, N. Mijajlovi, A second-order iterative method for solving quasi-variational inequalities, Comp. Math. Math. Phys. 53 (2013), 258-264.

[32] I.P. Ryazantseva, First-order methods for certain quasi-variational inequalities in a Hilbert space, Comput. Math. Math. Phys. 47 (2007), 183-190. 